\title{
Spectral Properties of Coupled Bose-Einstein Condensates
}

\author{
Roberto Franzosi and Vittorio Penna \\ Dipartimento di Fisica \&s Unità INFM, Politecnico di Torino, Corso Duca degli Abruzzi 24, I-10129 Torino, Italy.
}

(November 11, 2018)

\begin{abstract}
We investigate the energy spectrum structure of a system of two (identical) interacting bosonic wells occupied by $N$ bosons within the Schwinger realization of the angular momentum. This picture enables us to recognize the symmetry properties of the system Hamiltonian $H$ and to use them for characterizing the energy eigenstates. Also, it allows for the derivation of the single-boson picture which is shown to be the background picture naturally involved by the secular equation for $H$. After deriving the corresponding eigenvalue equation, we recast it in a recursive $N$-dependent form which suggests a way to generate the level doublets (characterizing the $H$ spectrum) via suitable inner parameters. Finally, we show how the presence of doublets in the spectrum allows to recover, in the classical limit, the symmetry breaking effect that characterizes the system classically.
\end{abstract}

\section{INTRODUCTION}

After the recent observation [1] of Bose-Einstein condensation in dilute atomic gases realized by confining a macroscopic population of atoms in a potential trap [2], a large amount of work has been devoted to construct devices where two condensates [3] are trapped in a double-well potential. The interaction of such coupled Bose-Einstein condensates (BEC) gives rise to quantum phenomena such as coherent tunneling and interference effects that have been the subject of a huge number of studies, both theoretic 腈] and experimental [5].

The present paper is focussed on the dynamical aspects inherent in the interaction of two condensates trapped in two identical wells. Such a dynamics has been studied thoroughly in Refs. [6] within the minimal interaction scheme

$$
\left\{\begin{array}{l}
i \hbar \dot{\psi}_{1}=\left[-\frac{\hbar^{2}}{2 m} \triangle-v+U\left|\psi_{1}\right|^{2}\right] \psi_{1}-T \psi_{2} \\
i \hbar \dot{\psi}_{2}=\left[-\frac{\hbar^{2}}{2 m} \triangle-v+U\left|\psi_{2}\right|^{2}\right] \psi_{2}-T \psi_{1}
\end{array}\right.
$$

where the classical fields $\psi_{j}(\mathbf{r}, t)$ obey two coupled GrossPitaevskii equations (GPE), and $U, v, T$, describe the interatomic scattering, the external potential and the tunneling amplitude, respectively. Fields $\psi_{j}(\mathbf{r})$ (often called the wave functions of the condensate [2]) are defined as the expectation value $\psi_{j}(\mathbf{r}, t)=\left\langle\hat{\Psi}_{j}(\mathbf{r}, t)\right\rangle$ of the field operators $\hat{\Psi}_{j}$ within the many-body quantum theory of BEC's [7]. The negligible space dependence of $\psi_{j}$ through the condensates allows one to set $\Delta \psi_{j} \simeq 0$ so that Eqs. (1) reduce to a hamiltonian system with two complex degrees of freedom where the nonlinear cubic terms provide the system with an ample variety of interwell processes.

Before discussing the goals of this paper, it is useful to briefly review the relevant traits of the dynamics issued from the space-independent form of Eqs. (11). Due to the assumption that the bosonic wells are identical, the asso- ciated model Hamiltonian $\mathcal{H}\left(\psi_{1}, \psi_{2}\right)=U\left(\left|\psi_{1}\right|^{4}+\left|\psi_{2}\right|^{4}\right)-$ $v \mathcal{N}-T\left(\psi_{1} \psi_{2}^{*}+\psi_{2} \psi_{1}^{*}\right)\left(\mathcal{N}=\left|\psi_{1}\right|^{2}+\left|\psi_{2}\right|^{2}\right)$ exhibits a permutational symmetry (PS) realized by the exchange of the dynamical variables $\psi_{1}, \psi_{2}$. Physically, this is represented by the population exchange, $\left|\psi_{j}\right|^{2}$ being the boson number of the $j$-th well up to a volume factor.

The structure of the model phase space $\mathcal{P}$ (this is twodimensional because $\mathcal{N}$ is a constant of motion) reflects in a nontrivial way the presence of the PS. For energies $E<E_{*},\left(E_{*}\right.$ is a critical value of the energy depending on the model parameters) the orbits are placed concentrically around the energy minimum, and cover a region $C_{0} \subset \mathcal{P}$ that has essentially the structure of the harmonic oscillator phase space. This can be shown to entail the oscillations of the two condensates' populations around the common value $\mathcal{N} / 2$. Also, for $E<E_{*}$ the PS maps each orbit in itself. The remaining part of $\mathcal{P}$, filled by orbits with $E>E_{*}$, is formed by two (spatially) disjoint components $C_{+}, C_{-}$such that $\mathcal{P} \equiv C_{+} \cup C_{-} \cup C_{0}$. In this case any given energy value $E$ is associated with two distinct orbits $\gamma_{+} \in C_{+}, \gamma_{-} \in C_{-}$such that $\gamma_{ \pm} \rightarrow \gamma_{\mp}$ under the PS action. The presence of two energy maxima (located in $C_{+}$and $C_{-}$symmetrically) causes such a structure. The remarkable feature is that, when $E$ crosses $E_{*}$ from below, the system undergoes a symmetry breaking (SB) phenomenon [8] (governed by a bifurcation mechanism [9]) since the system 'must' choose to evolve either along $\gamma_{+}$or along $\gamma_{-}$. Dynamically, this entails the emergence of a permanent gap between the condensates' populations. Such an effect is also called a self-trapping effect [6, 10] in that, within a finite range of $E$, the system never leaves the region $C_{+}\left(C_{-}\right)$where was initially placed to go in $C_{-}\left(C_{+}\right)$.

The investigation at the quantum level of the scenario just described has been performed in Ref. [10] through the model Hamiltonian

$$
H=U\left(n_{1}^{2}+n_{2}^{2}\right)-v N-T\left(a_{1} a_{2}^{+}+a_{2} a_{1}^{+}\right),
$$


which represents the quantum counterpart of the Hamiltonian $\mathcal{H}\left(\psi_{1}, \psi_{2}\right)$ for the Laplacian-free Eqs. (11). A simple way to obtain $H$ relies on the fact that for low numbers of bosons per well (a realizable experimental situation) one can replace the condensate wave functions $\psi_{j}$ 's with the raising (lowering) operator $a_{i},\left(a_{i}^{+}\right)$obeying the canonical commutators $\left[a_{i}, a_{j}^{+}\right]=\delta_{i j}, i=1,2$. Since $[N, H]=0$, the total number of bosons $N:=n_{1}+n_{2}$, $n_{i}:=a_{i}^{+} a_{i}$, is a constant of motion (to simplify, we shall denote its eigenvalue by $N$ as well). The rigorous derivation of $H$ is effected in Ref. [10] by using a mode dependent form of the field operator $\hat{\Psi}_{j}$ within the many-body quantum theory [1] of BEC's. It is important to recall as well that model (2) has been studied also in Ref. [8] from the viewpoint of dynamical system theory.

In this paper we investigate the spectral properties of model (2) by combining the use of the PS and of a further symmetry involving the change $T \rightarrow-T$. The latter will be called odd symmetry (OS) for recalling its basic role in determining the structure of the energy spectrum when the total boson number $N$ is odd. Such symmetries are used extensively to show that:

(i) after recovering the known nondegeneracy of the Hamiltonian spectrum (see Ref. [8, 10), each energy eigenstate is either symmetric or antisymmetric under the PS action,

(ii) the doublets (pairs of close energy levels occurring in the energy spectrum when the model parameters range in a suitable interval) always pair a symmetric eigenstates with an antisymmetric one; we shall show how this feature plays a basic role in the classic limit,

(iii) the separation mechanism causing the splitting of the energy levels (the splitting effect has been observed numerically in Refs. [8. 10]) can be explained in a purely analytic way,

(iv) the fact that total boson number is even/odd dramatically influences the eigenvalues' parity under the OS.

As to point (i), we wish to emphasize that the main consequence of the nondegeneracy is to prevent the SB phenomenon occurring in $\mathcal{P}$ as well as the ensuing selftrapping of the system on a specific orbit of the two ones associated with a given energy $E>E_{*}$. This reflects the intrinsic tunneling effect due to the quantum nature of the system.

For attaining results $(i)-(i v)$ we first reformulate the initial quantum problem of two coupled (identical) wells through the Schwinger realization [12] of the spin algebra in terms of two-boson operators. This allows one to reconstruct Hamiltonian (2) within its dynamical algebra (this is introduced in Appendix A). The form of $H$ thus obtained can be interpreted in terms of a bosonic model defined on a nonhomogeneous linear lattice with one effective boson (single-boson picture). Such a picture has been derived in Ref. [13] and is reviewed in Sec. II together with the related formal background. Thanks to its group-theoretic character such a formulation is appli- cable to many-well systems with any boson number $N$.

In Sec. III the diagonalization of $H$ is faced in a systematic way by making explicit the PS and OS action on the components of the energy eigenstates. The resulting characterization of the eigenstates leads to identify the recursive expression of the eigenvalue equation of $H$ both for even $N$ and for odd $N$. This, in turn, enables us to recognize explicitly a hidden parameter able to control in an analytic way the level distance of the doublets. Such a parameter seems to suggest an alternative procedure to evaluate perturbatively the energy levels. A similar mathematical problem was analysed in Ref. [8] as to the problem of the dynamical tunneling through a separatrix, where the splittings of the doublets were traced by using the standard perturbation method.

\section{SPIN PICTURE OF THE TWO-WELL MODEL}

A convenient way to study the spectrum of $H$ consists in reformulating $H$ by means of the Schwinger picture of spin operators. The latter is a two-boson realization of the spin operators 12]

$$
J_{1}=\frac{a_{1} a_{2}^{+}+a_{2} a_{1}^{+}}{2}, J_{2}=\frac{a_{1} a_{2}^{+}-a_{2} a_{1}^{+}}{2 i}, J_{3}=\frac{n_{2}-n_{1}}{2},
$$

satisfying the commutators $\left[J_{a}, J_{b}\right]=i \epsilon_{a b c} J_{c}$ of the algebra $\operatorname{su}(2)$, where $a, b, c=1,2,3$, and $\epsilon_{a b c}$ is the totally antisymmetric tensor [12]. The generators $J_{a}$ of $\mathrm{su}(2)$ commute with the Casimir operator $C \doteq J_{1}^{2}+J_{2}^{2}+J_{3}^{2} \equiv$ $J_{4}\left(J_{4}+1\right)$ which, in the Schwinger picture, leads to the identification $J_{4} \equiv\left(n_{2}+n_{1}\right) / 2$. Consistently, one can check that $\left[J_{4}, J_{a}\right] \equiv 0$. Therefore, $\mathrm{su}(2)$ can be used to rewrite Hamiltonian (2) which takes the nonlinear form

$$
H=2\left[U J_{4}^{2}-v J_{4}+U J_{3}^{2}-T J_{1}\right]
$$

The spin picture embodies explicitly in $H$ the dimension $(2 J+1)$ of the Hilbert space $\mathcal{H}(N)$ of physical states of $H$, where the eigenvalue $J(=N / 2)$ of $J_{4}$ is the index of the spin representation [12]. The standard basis $\mathcal{B}_{N}=\{|J, m\rangle,|m| \leq J=N / 2\}\left(J_{3}|J, m\rangle=m|J, m\rangle\right)$ of $\mathcal{H}(N)$ is related to the number operator states through $|J, m\rangle=\left|n_{1}, n_{2}\right\rangle$, where $J=\left(n_{2}+n_{1}\right) / 2, m=\left(n_{2}-\right.$ $\left.n_{1}\right) / 2$. Thus $H$ and $J_{a}$ can be seen as $(2 J+1) \times(2 J+1)$ matrices. The fact that $2 J_{4} \equiv N$ is a constant of motion due to $[H, N]=0$ is ensured by $\left[J_{4}, J_{a}\right] \equiv 0$.

An important consequence made explicit by the spin picture is that the nonlinear term $J_{3}^{2}$ arising in Eq. (3) prevents the standard procedure of diagonalization of $H$ via a unitary trasformation of the group $\mathrm{SU}(2)$. Such a procedure works only for matrices that can be written as linear combinations of the algebra generators in that, by construction, they can be reduced to one of the generators by some appropriate group transformations [12]. Since the diagonalization is greatly simplified by such a 
reduction, the latter practically identifies with the diagonalization itself. When a matrix $\mathcal{O}$ contains nonlinear terms of its generating algebra (as in the case of Hamiltonian (3) with respect to $\mathrm{su}(2)$ ), resorting to a larger algebraic structure $\mathcal{A}$ enables one to express $\mathcal{O}$ as a linear combination of generators of $\mathcal{A}$. Such an enlarged algebra is called a dynamical algebra [14] for $\mathcal{O}$.

About Hamiltonian $H$ the problem is solved by representing the algebra $\mathrm{su}_{N}(2)$ [subscript $N(=2 J)$ recalls the algebra link with the total boson number] as a subalgebra of $\mathcal{A}=\mathrm{su}(M)$ for a suitable values of $M$. In Appendix A we show that $\mathcal{A} \equiv \mathrm{su}_{1}(N+1)$. The realization in $\mathcal{A}$ of the $\mathrm{su}_{N}(2)$ generators occurring in $H$ reads

$J_{3}=\Sigma_{q}(J+1-q) n_{q}, J_{+}=\Sigma_{q}[q(2 J+1-q)]^{\frac{1}{2}} b_{q}^{+} b_{q+1}$,

$J_{-} \equiv\left(J_{+}\right)^{+}\left(J_{ \pm}=J_{1} \pm i J_{2}\right)$, where $b_{q}^{+}\left(b_{q}\right)$ are raising (lowering) bosonic operators, $n_{q}=b_{q}^{+} b_{q}$, and $q \in$ $[1, N+1]$. Recalling that the constraint $N_{b}:=\Sigma_{q} n_{q} \equiv 1$ must be accounted for (see Appendix A) we shall call the realization just obtained single-boson picture. This furnishes the simplest way to represent in a linear form the nonlinear term of $H$. In fact, Hamiltonian (3) becomes

$H=C+2 U \Sigma_{q}\left[m^{2}(q) n_{q}-\tau R(q, J)\left(b_{q}^{+} b_{q+1}+h . c.\right)\right]$,

with $\tau:=T / U, R(q, J):=\left[(J+1 / 2)^{2}-(m(q)-1 / 2)^{2}\right]^{1 / 2}$, $m(q):=J+1-q$, and $C=2\left[U J^{2}-v J\right]$, where the quadratic term has been re-expressed as a linear combination of number operators $n_{q}$. A valuable alternative form of the original model (2) is offered by Eq. (4) which recasts the two-well dynamics in terms of the dynamics of a single boson on a linear nonhomogeneous lattice. In the single-boson picture physical states are expressed as 15]

$$
|\Psi\rangle=\Sigma_{q} \psi_{q} b_{q}^{+}|0, \ldots, 0\rangle
$$

with the normalisation condition $\Sigma_{q}\left|\psi_{q}\right|^{2}=1$ (see Appendix A). The system dynamics thus takes place on a hypersphere inside $\mathbf{C}^{N+1}$, where it can be interpreted in a classical form. This matches the fact that states (5) can be shown to be $\mathrm{su}_{1}(N+1)$ coherent states [14]. Based on Eq. (5), the Schrödinger problem $i \partial_{t}|\Psi\rangle=H|\Psi\rangle$ can be rewritten as a set of equations of motion

$$
i \dot{\Psi}_{m}=2 U m^{2} \Psi_{m}-T\left[R_{m+1}^{(J)} \Psi_{m+1}+R_{m}^{(J)} \Psi_{m-1}\right],
$$

where we have introduced $\Psi_{m}:=\psi_{q}$, and $R_{m}^{(J)}:=R(q, J)$ with $m:=J+1-q$, to join the present formalism with the spin picture basis $\mathcal{B}_{N}$ where states are labeled by $m \in[-J, J]$. It is worth noting how Eqs. (6) can be derived as well from the effective Hamiltonian

$$
\langle H\rangle=C+\Sigma_{m}\left[2 U m^{2}\left|\Psi_{m}\right|^{2}-T R_{m}^{(J)}\left(\Psi_{m}^{*} \Psi_{m-1}+\text { c.c. }\right)\right],
$$

representing the energy expectation value $\langle\Psi|H| \Psi\rangle \equiv$ $\langle H\rangle$, provided the Poisson structure $\left\{\Psi_{m}, \Psi_{\ell}^{*}\right\}=\delta_{m \ell} / i \hbar$ is assumed (see commment [16]). The time evolution of the dynamical variables (the components of $|\Psi\rangle$ ) is determined once the initial condition $|\Psi(0)\rangle$ at $t=0$ has been assigned. Upon denoting by $X_{m}$ the components of the $H$ eigenstates $|X\rangle$, one can retrieve the secular equation

$$
E X_{m}=2 U m^{2} X_{m}-T\left[R_{m+1}^{(J)} X_{m+1}+R_{m}^{(J)} X_{m-1}\right],
$$

from Eq. (6). $X_{m}$ 's can be shown to be real.

The procedure relying on the dynamical algebra construction has led to interpret model (2) as a lattice model with one boson via Eq. (何. This procedure has been implemented as well for illustrative purposes since it shows clearly how the nonlinearity occurring in the matrix form of $H$ is transferred to the coefficients of the linear combination in $\mathcal{A}$. We point out that such a simplification also works for a linear chain of $S$ interacting wells with $N$ bosons whose Hamiltonian can be written via the generators of $\operatorname{su}_{N}(S)$. The latter, in fact, can be always immersed within an algebra $\operatorname{su}(M)$ with $M$ sufficiently larger than $S$; moreover the two-boson realization of $\operatorname{su}(M)$ can be obtained for any $M$.

The component form of secular equation (8) represents, at the operational level, a basic intermediate result. The latter, as shown in Sec. III, is used to characterize explicitly (in the same spirit of Bloch's theorem for electronic wave functions) the inner spectrum structure as well as the structure of the energy eigenstates.

\section{SPECTRUM STRUCTURE}

In order to investigate the spectrum structure, we consider first the effects of the PS and the OS on the energy eigenstates, and make explicit how such two symmetries strongly characterize the eigenstate components. Then, we employ the results of such an analysis to recast the eigenvalue equation related to equation (8) in a recursive form, and identify the parameters able to control the splitting of the energy levels. The case with half-integer $J$ and integer $J$ are treated separately.

The PS is realized via the action of the unitary transformation $U_{1}:=\exp \left[i \pi J_{1}\right]$ which takes $J_{3}=\left(n_{2}-n_{1}\right) / 2$ into $U_{1} J_{3} U_{1}^{+}=-J_{3}$. This matches the effect of the PS classical action which involves the exchange of populations $n_{1}$ and $n_{2}$. Let us introduce the hermitian operator $P:=\exp [-i \pi J] U_{1}$ whose action on the states $|m\rangle$ (we drop the representation index $J$ in $|J, m\rangle$ since it is fixed) is deducible from the equation $U_{1}|m\rangle=\exp [i \pi J]|-m\rangle$. Owing to $\left[H, U_{1}\right]=0, P$ can be diagonalized together with $H$. The action of $P$ on the standard basis, $P|m\rangle=$ $|-m\rangle$, implies that, for a generic state $|\Psi\rangle$,

$$
P:|\Psi\rangle=\Sigma_{m} \Psi_{m}|m\rangle \rightarrow P|\Psi\rangle=\Sigma_{m} \Psi_{m}|-m\rangle .
$$

In particular, the $P$ action on an eigenstate $|X\rangle$ entails the component transformation $X_{m} \rightarrow \sigma X_{-m}$, where $\sigma$ is not fixed, in Eqs. (8). Actually, these remain unchanged 
since $R_{m+1}^{(J)} \equiv R_{-m}^{(J)}$ for each $m$. The fact that $P^{2} \equiv \mathbf{I}$ fixes $\sigma$ showing how the allowed eigenvalues for $P$ are $\sigma= \pm 1$. Since $P: X_{m} \rightarrow \pm X_{-m}$, each eigenstate has thus a definite symmetry character under $m \rightarrow-m$.

This fact suggests to reorganize the vectors basis in terms of vectors $|m, \pm\rangle=(|m\rangle \pm|-m\rangle) / \sqrt{2}$ that are either symmetric or antisymmetric. The new basis allows one to define in $\mathcal{H}(N)$ a symmetric (antisymmetric) subspace $\mathcal{H}^{+}(N)\left(\mathcal{H}^{-}(N)\right)$ whose vectors have components we will denote by $\Phi^{+}\left(\Phi^{-}\right)$such that $P \Phi^{ \pm}= \pm \Phi^{ \pm}$. When the description in the new basis is adopted then Eqs. (8) for the eigenvector components can be written in the matrix form

$$
E\left[\begin{array}{c}
X^{+} \\
X^{-}
\end{array}\right]=\left[\begin{array}{c|c}
S_{J}(T) & 0 \\
\hline 0 & A_{J}(T)
\end{array}\right]\left[\begin{array}{c}
X^{+} \\
X^{-}
\end{array}\right]
$$

where $S_{J}(T)\left(A_{J}(T)\right)$ is the sub-matrix associated with symmetric (antisymmetric) sector, and 0 represents the zero-matrix. The matrix equation (9) is separable in two independent equations for $X^{+}$and $X^{-}$: Their explicit form which depends on the representation index $J$ is displayed in the sequel.

The odd symmetry (denoted by OS) is obtained by combining the action $U_{3} J_{1} U_{3}^{+}=-J_{1}$ of $U_{3}:=\exp \left[i \pi J_{3}\right]$ on $-T J_{1}$ in $H$ with the change $T \rightarrow-T$ which restores the initial form of $H$. Since $J$ can be either integer or half-integer, for considering the two cases separately it is convinient to introduce the matrix $L_{J}(T):=\left\|L_{m \ell}\right\|$

$$
L_{m \ell}=2 U m^{2} \delta_{m, \ell}-T\left[R_{m}^{(J)} \delta_{m, \ell+1}+R_{\ell}^{(J)} \delta_{m+1, \ell}\right],
$$

where $m, \ell=1 / 2,3 / 2, \ldots, J$ if $J$ is half-integer, and $m, \ell=1,2, \ldots, J$ if $J$ is integer.

Let us start with the half-integer case. In Eq. (9), the sub-matrices $S_{J}(T)$ and $A_{J}(T)$ coincide with the matrix $L_{J}(T)$ up to the quantity $-\eta T R_{1 / 2}^{(J)}$ which must be added to the matrix element $L_{\frac{1}{2} \frac{1}{2}}$ with $\eta=-1(+1)$ in the antisymmetric (symmetric) case. Representing $U_{3}$ in the basis $(\{|m, \pm\rangle\})$ entails

$$
U_{3}=\left[\begin{array}{c|c}
0 & D \\
\hline-D & 0
\end{array}\right]
$$

in which $D=\operatorname{Diag}(i,-i, \ldots)$. The action of $U_{3}$ on any vector takes its symmetric components into the antisymmetric ones and vice versa, namely $P U_{3} \Phi^{ \pm}=\mp U_{3} \Phi^{ \pm}$ if $P \Phi^{ \pm}= \pm \Phi^{ \pm}$. The structure of energy spectrum is reconstructed through the following three observations: i) The secular equation for $X^{+}$and $X^{-}$derived from Eqs. (9) can be written in the reduced form

$$
E C_{m}=2 U m^{2} C_{m}-T\left[R_{m+1}^{(J)} C_{m+1}+R_{m}^{(J)} C_{m-1}\right],
$$

with $C=X^{+}, X^{-}$, for $1 / 2<m \leq J$, whereas

$$
0=\left[2 U(1 / 2)^{2}-\eta T R_{1 / 2}^{(J)}-E\right] C_{1 / 2}-T R_{3 / 2}^{(J)} C_{3 / 2},
$$

holds for $m=1 / 2$, where $\eta \equiv-1(+1)$ in the antisymmetric (symmetric) case. For a given $T \neq 0$, Eqs. (12) and (13) show that the eigenvalues $E$ can be seen as a set of functions $E_{a}(T, \eta)$ of $\eta$ with $1 / 2 \leq a \leq N / 2$, defined implicitly. The energy eigenvalue $E_{a}(T,+1)$ of each symmetric eigenstate can be derived from that $E_{a}(T,-1)$ of an antisymmetric eigenstate by moving $\eta$ from -1 to +1 , and vice versa.

ii) We consider here the problem of ordering the set of eigenvalues $E_{a}(T, \pm 1)$. For $T \rightarrow 0$ (this eliminates the $\eta$ dependence) Eqs. (8) are solvable; the resulting eigenvalues are doubly degenerate since the equations for both the symmetric and the antisymmetric components are identical. Explicitly, $T \rightarrow 0$ entails $E_{a}(T,+1), E_{a}(T,-1) \rightarrow 2 U m^{2}$ for some $m$ which shows how the label $a$ can be identified with $m \in[1 / 2, J]$. The order induced by (positive) $m$ on the set $\left\{2 U m^{2}\right.$ : $|m| \leq J\}$ is inherited both by the symmetric eigenvalues $\left\{E_{a}(T,+1)\right\}$ and by the antisymmetric eigenvalues $\left\{E_{a}(T,-1)\right\}$ as proven by the limit $T \rightarrow 0$. This also implies that, for sufficiently small $T, E_{a}(T, \pm 1) \neq$ $E_{b}(T, \pm 1)$ if $a \neq b$.

iii) Implementing the action of $U_{3}$ whose matrix form is given by Eq. (11) on Eq. (9) leads to the equation

$$
E\left[\begin{array}{c}
\tilde{X}^{-} \\
\tilde{X}^{+}
\end{array}\right]=\left[\begin{array}{c|c}
A_{J}(-T) & 0 \\
\hline 0 & S_{J}(-T)
\end{array}\right]\left[\begin{array}{c}
\tilde{X}^{-} \\
\tilde{X}^{+}
\end{array}\right],
$$

where $\tilde{X}^{\mp}=D X^{ \pm}$, and $P \tilde{X}^{ \pm}= \pm \tilde{X}^{ \pm}$. The substitution $T \rightarrow-T$ entails that [we use the simplified notation $\left.E_{a}^{ \pm}(T):=E_{a}(T, \pm 1)\right]$ the set of the symmetric (antisymmetric) eigenvalues $\left\{E_{a}^{+}(T)\right\} \quad\left(\left\{E_{a}^{-}(T)\right\}\right)$ coincides with the set of the antisymmetric (symmetric) ones $\left\{E_{b}^{-}(-T)\right\}\left(\left\{E_{b}^{+}(-T)\right\}\right)$, where $1 / 2 \leq a, b \leq J$. Notice that, in general, $E_{a}^{ \pm}(T) \equiv E_{b}^{\mp}(-T)$, where not necessarily $b$ coincides with $a$. Nevertheless, for $T \rightarrow 0$ $E_{a}^{ \pm}(0) \equiv E_{b}^{\mp}(0) \equiv 2 U a^{2}=2 U b^{2}$ implies that $b=a$, as pointed out at point (ii).

As a consequence of points (i)-(iii), we find that the symmetric eigenvalues are associated with the antisymmetric ones through the formula

$$
E_{a}^{ \pm}(T)=E_{a}^{\mp}(-T) \quad(1 / 2 \leq a \leq J) .
$$

Also, since the eigenvalues equation can be cast in an iterative form via the recurrence equation

$$
d_{m}(E)=\left(2 U m^{2}-E\right) d_{m+1}(E)-T^{2}\left[R_{m+1}^{(J)}\right]^{2} d_{m+2}(E),
$$

which starts from

$0=\left[U / 2-E+\eta T R_{1 / 2}^{(J)}\right] d_{\frac{3}{2}}(E)-T^{2}\left[R_{1 / 2}^{(J)}\right]^{2} d_{\frac{5}{2}}(E)$,

and terminates with $d_{J}(E)=2 U J^{2}-E$, consistently with (iii) one finds that the eigenvalues cannot be even functions of $T$. 
For integer $J$, the dimension of matrix $S_{J}(T)$ changes from that of matrix $A_{J}(T)$. In the antisymmetric case one finds $A_{J}(T)=L_{J}(T)$, while in the symmetric case $\left(S_{J}(T):=\left\|S_{m n}\right\|\right)$, where the indices runs over $0,1, \ldots, J$, one finds $S_{01}=S_{10}=-T R_{1}^{(J)}, S_{m n}=L_{m n}$ for $m, n \geq 1$, and $S_{m n}=0$ otherwise. Eqs. (12) still hold for integer $J$ provided $2 \leq m \leq J$. The two special cases are those corresponding to $m=0,1$

$$
\begin{gathered}
0=-E C_{0}-T \sigma R_{1}^{(J)} C_{1}, \\
0=(2 U-E) C_{1}-T\left[R_{2}^{(J)} C_{2}+\sigma R_{0}^{(J)} C_{0}\right],
\end{gathered}
$$

with $C=X^{+}, X^{-}$. The parameter $\sigma$ must be set equal to one in the symmetric case $\left(C=X^{+}\right)$, while in the antisymmetric case $\left(C=X^{-}\right)$the expected elimination of the component $X_{0}^{-}$follows from setting $\sigma=0$. Hence the dimensions of Hilbert sub-spaces are such that $\operatorname{dim} \mathcal{H}^{-}(N)=\operatorname{dim} \mathcal{H}^{+}(N)-1$, while the secular equation for $X^{-}$will have a degree diminished of one. Explicitly, one has

$0=\left[\frac{E(2 U-E)}{T^{2}}+\sigma^{2}\left[R_{1}^{(J)}\right]^{2}\right] d_{2}(E)-E\left[R_{2}^{(J)}\right]^{2} d_{3}(E)$.

In the symmetric case a $(J+1)$-th degree equation for $E$ issues from (20) through formula (16). Comparing the eigenvalue equations for the symmetric $(\sigma=1)$ and antisymmetric $(\sigma=0)$ states shows that each, but one, symmetric eigenvalue merges to an antisymmetric one when $\sigma$ goes from 0 to +1 . Due to the diversity of the secular equation with $\sigma=1$ from that with $\sigma=0$, even in the case with integer $J$, the energy spectrum is constituted by $2 J$ nondegenerate eigenvalues $\left\{E_{a}^{ \pm}(T): 1 \leq a \leq J\right\}$ that for $T \rightarrow 0$ form $J$ pairs $E_{a}^{ \pm}(T) \rightarrow 2 U a^{2}$ and a single one $E_{0}(T)$ which goes to zero in the same limit. Also, due to the quadratic dependence of Eqs. (16), (20) on $T$, Eq. (15) must be replaced with

$$
E_{a}^{ \pm}(T)=E_{a}^{ \pm}(-T) \quad(1 \leq a \leq J),
$$

which, contrary to what happens with half-integer $J$, maps each eigenvalue in itself under $T \rightarrow-T$. In addition, of course, one must consider $E_{0}(T)=E_{0}(-T)$ as well. Figs. 1 illustrate the spectrum structure dependence on $T / U$ for $N=6,7$ (see also Fig. 2).

\section{DISCUSSION}

The interesting feature disclosed by the above analysis is the possibility to recognize both in the half-integer and in the integer case two inner parameters $(\eta$ and $\sigma$ ) that control, in a way independent of $T$, the level splitting generating the doublets. The limit $T \rightarrow 0$ causes a coalescence of doublet levels such that $E_{a}^{ \pm}(T) \rightarrow 2 U a^{2}$ which suggests $T$ as a possible perturbative parameter for evaluating the level splitting. On the other hand, Fig. 2 clearly shows that each eigenvalue $E_{a}^{+}(T)$ remains close to its partner $E_{a}^{-}(T)$ on a finite range $I_{a}(T)$ of $T$ indexed by the eigenvalue label $a$. In the half-integer case, this implies that, inside $I_{a}(T)$, indeed $\eta$ represents a good perturbative parameter (recall that $E_{a}^{ \pm}(T)=E_{a}(T, \eta)$ with $\eta= \pm 1)$ which allows one to evaluate $E_{a}(T, \pm 1)$ by perturbing $E_{a}(T, \eta)$, e. g., around $\eta=0$. For integer $J$, where the level separation is controlled by $\sigma \in[0,1]$, one can observe an effect similar to that showed in Fig. 2: the symmetric eigenvalue $E_{a}(T, 1)=E_{a}^{+}(T)$ remains close to its antisymmetric partner $E_{a}(T, 0)=E_{a}^{-}(T)$ on a finite range. Because the function $E_{a}(T, \sigma)$ joins analitically $E_{a}^{-}(T)$ to $E_{a}^{+}(T)$ then $\sigma$ can be assumed as the perturbative parameter for the present case.

The actual size of the range $I_{a}(T)$ can be evinced roughly from Fig. 2, where the level separation strongly diverges only when $E_{a}^{ \pm}(T)$ cross $E \equiv E_{*}:=N T$ (recall that $E_{*}$ is the energy critical value defined in the introduction; its derivation can be found in Ref. 13i|). This rule seems to be motivated from the insensitivity of Eq. (17) (Eq. (20)) from the parameter $\eta(\sigma)$ in the terms

$$
\eta T R_{1 / 2}^{(J)}-E \quad\left(\sigma^{2}\left[R_{1}^{(J)}\right]^{2}-E^{2} / T^{2}\right),
$$

for suitable values of $E, T$ and of the other coefficients.

Concerning the classical limit effected through $N \rightarrow$ $\infty$, numerical simulations with large boson numbers $N$ show that the series of doublets becomes degenerate (coalescence of the doublet levels) thus restoring the conditions that allow for the SB effect. How recovering the latter is briefly illustrated via the following comparison between the classical and the quantum behavior of the twowell system. Classically, at a given energy $E>E_{*}$, the system described in $\mathcal{P}$ evolves either on $\gamma_{-}$or on $\gamma_{+}$(see Sec. I). Trajectories $\gamma_{-}$and $\gamma_{+}$are such that the populations' gap $n_{2}-n_{1}$ weakly oscillates around opposite values $-\mu$ and $+\mu$, respectively. Quantally, for $E>E_{*}$, the combination $\left|C_{a}^{ \pm}\right\rangle=\left|X_{a}^{+}\right\rangle \pm\left|X_{a}^{-}\right\rangle$of the symmetric/antisymmetric eigenstates $\left|X_{a}^{ \pm}\right\rangle$of the ath doublet can be shown to provide opposite nonvanishing expectation values $\left\langle J_{3}\right\rangle= \pm \chi$ of $J_{3}=\left(n_{2}-n_{1}\right) / 2$. This fact is caused by the eigenstate structure and was discussed in Ref. [13]. Then, states $\left|C_{a}^{ \pm}\right\rangle$can be associated naturally to a pair of isoenergetic orbits $\gamma_{-}, \gamma_{+}$that have $\mu \equiv 2 \chi$. Increasing $N$, the time-dependent mixed state

$$
|\Psi\rangle=e^{-i t E_{a}^{+}(T) / \hbar}\left|X_{a}^{+}\right\rangle+e^{-i t E_{a}^{-}(T) / \hbar}\left|X_{a}^{-}\right\rangle
$$

(satisfying the Schrödinger problem of $H$ ) exhibits a sort of temporary self-trapping effect (i. e. the localization either on $\gamma_{-}$or on $\gamma_{+}$) which is repeated periodically and has a duration increasing with $N$. In fact, because of the oscillations of the factor $\exp \left\{\left[i t\left(E_{a}^{+}(T)-E_{a}^{-}(T)\right]\right\}\right.$ between +1 and -1 entailing $|\Psi\rangle \propto\left|C_{a}^{+}\right\rangle$and $|\Psi\rangle \propto\left|C_{a}^{-}\right\rangle$, respectively, the system stays in a quantum state involving the localization on $\gamma_{ \pm}$for intervals of the order of 
the period $\Delta t=2 \pi \hbar /\left[E_{a}^{+}(T)-E_{a}^{-}(T)\right]$ that increase when the level separation is reduced. The system remains definitively in the classical-like states (full emergence of the SB effect inducing the self-trapping) when the tunneling time from $\left|C_{a}^{+}\right\rangle$to $\left|C_{a}^{-}\right\rangle$diverges, namely for $\left[E_{a}^{+}(T)-E_{a}^{-}(T)\right] \rightarrow 0$ (coalescence of doublet levels induced by $N \rightarrow \infty)$.

\section{CONCLUSIONS}

In Sec. I we have reviewed the dynamics of coupled Bose condensates described by Eqs. (11) (in the approximation with zero Laplacian terms) emphasizing the SB phenomenon that occurs in the phase space when increasing the energy over the critical value $E_{*}$. Such a phenomenon (and the ensuing self-trapping effect on isoenergetic orbits $\gamma_{ \pm} \in \mathcal{P}$ where $n_{2}-n_{1}$ oscillates around nonzero values $\pm \mu$ ) has prompted the study of model (2) which represents the quantum counterpart of model (1) within the two-mode approximation of the condensate field operator. One of the purposes of the present work was to investigate the dynamical behavior corresponding, at the quantum level [through model (2)], both to the SB effect and to the related self-trapping. An aspect we have particularly deepened is the quantum counterpart (level splitting) of the bifurcation mechanism generating pair of isoenergetic trajectories $\gamma_{ \pm}$when $E$ crosses $E_{*}$.

The formal set-up for studying the energy spectrum has been developed in Sec. II by recasting model (2) for the boson modes $a_{1}, a_{2}$ into the matrix form (3) within the spin formulation $\grave{a}$ la Schwinger. The spin form of Hamiltonian (3) makes easily viable the derivation of the secular equation (8). The latter has been achieved by using the dynamical algebra method (this enacts systematically the reduction of Hamiltonian nonlinearities) whose application is described in Appendix A.

In addition to supplying equation (8), the use of the dynamical algebra method has shown the implicit link of the secular equation with the effective-bosons model (位). Such a model reformulates the two-well dynamics of Hamiltonian $H$ with $N$ bosons in a noticeably simplified form that consists of a single boson hopping on a nonhomogenous lattice (single boson picture). The interest for the single boson picture and the underlying formal construction is motivated by the possibility of extending it to more structured models such as a chain model of $S$ condensates with $N$ boson. The case $S=3$, which raises interest owing to its nonintegrable dynamical character, is presently under investigation 18 .

Sec. III has been devoted to make explicit the structure of the energy spectrum based on the symmetries of $H$. Upon introducing the permutational symmetry (PS) and recognizing the further odd symmetry (OS), we have employed them to characterize the Hilbert space of $H$ as well as the energy eigenstates, both for half-integer $J$ (odd number of boson $N$ ) and for integer $J$ (even number of boson $N$ ). The $N$-dependent form of the secular equation obtained in the two cases has led to the central result of Sec. III, namely to recognize the possibility of introducing in a natural way inner parameters that control the level splitting in the energy spectrum doublets.

Such parameters $-\eta[\sigma]$ is defined in Eq. (17) [Eq. (20)] for half-integer [integer] $J$-have shown that the splitting originating the doublets can be generated explicitly in an analytic way. This fact combined with the doublets' structure exhibited in Fig. (2) suggests that parameters $\eta, \sigma$ can be used as perturbative parameters in approximating the doublet levels inside the regions of the $T / U$ axis where the levels keep close. This approach may be preferable than the standard perturbative approach depending on the natural parameter $T / U$ : this, in fact, can be shown to require higher and higher powers of $T / U$ when approximating levels far from the ground-state [8].

We emphasize the fact that generating the level splitting via the inner parameters $\eta, \sigma$ can be interpreted as the quantum form of the bifurcation mechanism issuing pairs of orbits $\gamma_{ \pm}$. In general, our construction should furnish the quantum framework for describing the bifurcation effects of any system whose Hamiltonian (in the critical regions of its phase space) has locally the same form of $H$. In this sense, both the quantum phase models and spin models [17] in the mesoscopic system physics promise interesting applications. We notice as well that the matrix/algebraic analysis underlying the study of 'quantum' bifurcation effects gives a valuable, both formal and practical, tool for characterizing quantally the chaos onset in the model with $S=3$.

The classical limit $N \rightarrow \infty$ has been commented in Sec. IV, where the symmetry breaking (SB) effect inherent in the classical two-well dynamics is recovered from the quantum scenario via superposition (22) of the symmetric and antisymmetric states of each doublet. In fact, the coalescence of the doublet levels caused by $N \rightarrow \infty$ (revealed by numerical simulations) leads, through a limiting process, to inhibit the oscillations of the system state $|\Psi\rangle$ between $\left|C_{a}^{+}\right\rangle$and $\left|C_{a}^{-}\right\rangle$which ends up by coinciding with one of such states. This realizes the localization interpreted classically as the self-trapping effect.

We conclude by illustrating a possible reformulation of Eq. (8) in a continuous form valid for large $J$ directed to extimate the low part of energy spectrum. Setting $X_{m} \equiv Y_{m} /[(m+J) !(J-m) !]^{1 / 2}$ in Eq. (8) provides $\left(2 U m^{2}-E\right) Y_{m}=T\left[(J-m) Y_{m+1}+(J+m) Y_{m-1}\right]$, which reduces to the second order equation $(\dot{F}:=d F / d x)$

$$
\ddot{F}-2 x s \dot{F}+(2 J+1-s R+E / T) F / R=0,
$$

where $s:= \pm 1$ and $x:=m / \sqrt{J R}$ with $R^{2}:=1+2 U J / T$, while $Y(m) \equiv \exp \left[-\alpha m^{2} / J\right] F(m / \sqrt{J R})$, with $\alpha:=$ $(s R-1) / 2$, is the dependence of the (rescaled) components $Y_{m}$ on (the continuous variable) $m$. The assumption that $F$ identifies with the $n$-th Hermite polynomial 
gives the equation $2 n \equiv[2 J+1-R+E / T] / R$ for $s=+1$ which, in turn, supplies a set of eigenvalues. Their dependence on $T$ is compared in Fig. 3 with the lowest part of the spectrum of $N=20$ bosons. This result as well as the results/observations discussed above have prompted further work that is presently in progress.

\section{ACKNOWLEDGMENTS}

We are indebted to E. Guadagnini and M. Rasetti for stimulating discussions. The financial support of I.N.F.M. (Italy) and of M.U.R.S.T. (within the Project Sintesi) is gratefully acknowledged.

\section{APPENDIX A:}

The type of enlarged algebra we deal with is $\mathcal{A}=$ $\mathrm{su}(M)$ [the latter can be viewed as the generalized version of the spin algebra with $M^{2}-1$ generators]. Selecting an appropriate value of $M$ allows one to rewrite the nonlinear Hamiltonian $H$ in terms of a linear combination of generators of $\mathcal{A}$. This furnishes $\mathcal{A}$ with the status of dynamical algebra for $H$. To construct explicitly $\mathcal{A}$ it is useful to consider the two-boson form of the $\mathrm{su}(\mathrm{M})$ generators $E_{i j}:=b_{i}^{+} b_{j},(i \neq j), H_{i j}:=\left(b_{i}^{+} b_{i}-b_{j}^{+} b_{j}\right) / 2$ that satisfy the commutators 12

$$
\begin{gathered}
{\left[E_{i j}, E_{k l}\right]=\delta_{j k} E_{i l}-\delta_{i l} E_{k j},} \\
{\left[E_{i j}, H_{k l}\right]=\left(\delta_{j k} E_{i k}-\delta_{j l} E_{i l}+\delta_{i l} E_{l j}-\delta_{i k} E_{k j}\right) / 2,}
\end{gathered}
$$

with $1 \leq i, j, l, k \leq M$. Within the present realization of $\operatorname{su}(M)$, the representation theory of semi-simple Lie groups [14] states that the eigenvalue $Q$ of the invariant operator $N_{b}=\Sigma_{i} b_{i}^{+} b_{i}\left(\left[N_{b}, E_{i j}\right]=0\right)$ selects a specific representation of $\operatorname{su}(M)\left[N_{b}\right.$ can be viewed as the total particle number relatively to the creation (destruction) processes caused by $b_{i}^{+}\left(b_{i}\right)$ ]. In fact, the dimension of the Hilbert space basis $B(M, Q)=\left\{\left|n_{1}, \ldots, n_{M}\right\rangle, Q=\right.$ $\left.\sum_{i=1}^{M} n_{i}\right\}$ is given by

$$
\operatorname{dim} B(M, Q)=(Q+M-1) ! /[(M-1) ! Q !],
$$

where the states of the basis $B(M, Q)$ are defined as $\left|n_{1}, \ldots, n_{M}\right\rangle=\otimes_{i=1}^{M}\left|n_{i}\right\rangle$ and the number operator states $\left|n_{j}\right\rangle$ fulfil the equations

$$
b_{i}\left|n_{i}\right\rangle=\sqrt{n_{i}}\left|n_{i}-1\right\rangle, b_{i}^{+}\left|n_{i}\right\rangle=\sqrt{n_{i}+1}\left|n_{i}+1\right\rangle .
$$

The simplest realization of $\mathcal{A}=\mathrm{su}_{Q}(M)$ is achieved by setting $Q=1$ (single-boson picture); combining this fact with the requirement of preserving the dimension $N+1$ of $\mathcal{H}(N)$, entails $\operatorname{dim} B(M, 1)=M \equiv N+1$ thus providing $\mathcal{A}=\mathrm{su}_{1}(N+1)$ as a dynamical algebra for $H$. The states of the related basis $\left\{|q\rangle=b_{q}^{+}|0, \ldots, 0\rangle, q \in[1, N+1]\right\}$ are in a one-to-one correspondence with the states $|J ; m\rangle$, $|m| \leq J=N / 2$, of the $\operatorname{su}_{N}(2)$ standard basis $\left(J_{3}|J ; m\rangle=\right.$ $m|J ; m\rangle)$ via the index map $m=J+1-q$.

[1] M. H. Anderson, J. R. Ensher, M. R. Matthews, C. E. Wieman, and E. A. Cornell, Science 269, 198 (1995). C. C. Bradley, C. A. Sackett, J. J. Tollett, and R. G. Hulet, Phys. Rev. Lett. 75, 1687 (1995).

[2] F. Dalfovo, S. Giorgini, Lev P. Pitaevskii, S. Stringari, Rev. Mod. Phys. 71, 463 (1999).

[3] K. B. Davis, M.-O. Mewes, M. R. Andrews, N. J. van Druten, D. S. Durfee, D. M. Kurn, and W. Ketterle Phys. Rev. Lett. 75, 3969 (1995);

[4] P. Öhberg and S. Stenholm, Phys. Rev. A 57, 1272 (1998); T. Esslinger, I. Bloch and T. W. Hänsch, Phys. Rev. B 58, 1480 (1998)

[5] M.R. Andrews et. al., Science 275, 637 (1997).

[6] A. Smerzi, S. Fantoni, S. Giovannazzi and S. Shenoy Phys. Rev. Lett. 79 , 4950 (1997); S. Raghavan, A. Smerzi, S. Fantoni, and S. Shenoy, Phys. Rev. A 59, 620 (1999).

[7] Another way to define $\psi_{j}(\mathbf{r}, t)$ derives from interpreting $\left|\psi_{j}\right|^{2}$ as the $j$-condensate density of bosons occupying the single-particle state with zero momentum (see Ref. [2]).

[8] S. Aubry, S. Flach, K. Kladko, and E. Olbrich, Phys. Rev. Lett. 76, 1607 (1996).

[9] Geometrically, one can say that $\mathcal{P}$ exhibits a bifurcation of its periodic orbits and a separatrix manifold that splits it into three regions: $C_{0}$ with orbits invariant under the PS, and $C_{ \pm}$whose (noninvariant) orbits are in ono-to-one correspondence via the PS action.

[10] G. J. Milburn, J. Corney, E. M. Wright and D. F. Walls, Phys. Rev. B 55, 4318 (1997).

[11] Using the two-mode expansion $\hat{\Psi}_{j}(\mathbf{r}, t) \simeq a_{1} u_{1}(\mathbf{r})+$ $a_{2} u_{2}(\mathbf{r})$ (the time is now embodied in $a_{j}$ 's) the distribution in two wells of original condensate described by $\hat{\Psi}_{j}$ is effected by the local modes $u_{j}(\mathbf{r})$ 's that make explicit the underlying double-well potential structure.

[12] W.-M. Zhang, D.H. Feng, and R. Gilmore, Rev. Mod. Phys. 62, 867 (1990).

[13] R. Franzosi, V. Penna and R. Zecchina, Int. J. Mod. Phys. B 14, 943 (2000).

[14] A.M. Perelomov, Generalized Coherent States and their Applications, (Springer Verlag, Berlin, 1986)

[15] It is important to recall that the system dynamics is embodied in the components $\psi_{q}$. The boson operators $b_{q}^{+}$, $b_{q}$ as well as the basis $\left\{b_{q}^{+}|0, \ldots, 0\rangle\right\}$ have a purely formal role involved by the enlargement of the algebra and of the Hilbert space thereof.

[16] The proper frequencies associated with Eq. (7) and the related periodic orbits coincide with the eigenvalues and the eigenstates of Eq. (6), respectively.

[17] L. Amico and V. Penna, Phys. Rev. B 62, 1224 (2000).

[18] R. Franzosi, and V. Penna in preparation. 
FIG. 1. First figure shows the energy eigenvalues vs. $T / U$ for $J=7 / 2$; second figure shows the case $J=3$ (different types of line identify different eigenvalues).

FIG. 2. Solid lines represent the eigenvalues $E_{a}^{ \pm}$vs. $T / U$ for $J=15 / 2$. The dashed line describes the curve $E=2 J T$. For each pair $E_{a}^{ \pm}$, the splitting becomes evident when $2 J T$ reachs $E_{a}^{ \pm}$.

FIG. 3. The figure shows the seven lowest eigenvalues vs. $T / U$ for $J=10$ (solid lines); the seven dashed lines corrispond to the approximate eigenvalues derived from Eq. (23) for $s=+1$. The approximation remains good for large values of $T / U$. 